\title{
The influence of circadian variation in ischemic stroke onset on the evolution of the severity of the clinical picture and disability
}

\author{
Dana Marieta Fodor' ${ }^{1}$, Marius Marian Fodor ${ }^{2}$, Ioana Cristina Stănescu' ${ }^{1}$, Gabriela Dogaru ${ }^{3}$, \\ Lăcrămioara Perju-Dumbravă ${ }^{1}$
}

Corresponding author: Marius Marian Fodor, E-mail: drfodor@yahoo.com,

1.Neuroscience Department, "Iuliu Haţieganu" University of Medicine and Pharmacy, Cluj-Napoca, Romania 2.Vascular Surgery Department, Emergency County Hospital, Cluj-Napoca, Romania 3."Iuliu Hatieganu" University of Medicine and Pharmacy, Cluj-Napoca, Clinical Rehabilitation Hospital, Cluj-Napoca, Romania

\begin{abstract}
Introduction. The chronobiology of ischemic stroke describes an occurrence pattern with the highest incidence in the morning according to most literature reports, but its influence on the evolution of the severity of the neurological picture and functional status is little studied. Materials and method. This cohort study included 63 patients with ischemic stroke admitted to the Neurology Departments I and II of the Rehabilitation Hospital in Cluj-Napoca between 1 June 2008 and 1 June 2009, who were followed up for 2 years by 5 successive evaluations. The onset time of ischemic stroke was assigned to one of the six hours intervals: 00.0106.00 (night), 06.01-12.00 (morning), 12.01-18.00 (afternoon) and 18.01-24.00 (evening). For each patient, the National Institute of Health Stroke Scale (NIHSS) and Modified Rankin Scale (mRS) scores were recorded on the occasion of each evaluation. Statistical analysis was performed using Excel Microsoft, descriptive and ANOVA test. Results and conclusions. Our study confirms the incidence pattern of ischemic stroke with a morning peak, which is more obvious in the case of patients aged less than 65 years. Patients with stroke onset in the nocturnal interval have a less favorable neurological and functional evolution during the second year after ischemic stroke.
\end{abstract}

Key words: ischemic stroke occurrence, circadian variation, NIHSS, $m R S$,

\section{Introduction}

Over the past years, interest has increased in the study of chronobiological aspects involved in health maintenance as well as in the development of various diseases. The study of the temporal variation in the incidence of cerebrovascular pathology is part of this interest (1-4). Ischemic stroke describes a circadian variation pattern with the highest incidence in the morning, according to most literature reports (5-9). The NIHSS Scale (the National Institute of Health Stroke Scale) reflects the severity of the neurological picture, and the mRS disability score (Modified Rankin Score) assesses the degree of functional impairment in post-ischemic stroke patients, both scores representing parameters that allow monitoring patient evolution (10). The evolution of the severity of the neurological picture and disability after ischemic stroke depending on the time of the day when ischemic stroke has occurred is little studied (11-13).

\section{Materials and method}

A prospective cohort study was conducted which included 63 patients with ischemic stroke occurring over the last 6 months, admitted to the Neurology Departments I and II of the Rehabilitation Hospital in Cluj-Napoca, in the period 1 June 2008 - 1 June 2009. The diagnosis of ischemic stroke was defined according to updated World Health Organization criteria and was confirmed by neuroimaging. For each patient, demographic data were recorded and the time of onset was assigned to one of the 4 intervals of the day: 00.01-06.00 (night), 06.01-12.00 (morning), 12.01-18.00 (afternoon) and 18.01-24.00 (evening).

The 63 patients were followed up on the occasion of 5 visits during 2 years: first visit (time " 0 "), at 1 month ("1"), 6 months ("6"), 12 months ("12"), and 24 months ("24"). Each patient was assessed based on the NIHSS and mRS scores. Four of these patients missed one visit. 
Statistical analysis was performed using Excel Microsoft software. Categorical data were presented as diagrams, absolute and relative frequencies, and continuous variables were summarized using synthetic centrality, dispersion and location indicators or frequency histograms and linear diagrams. For the analysis of the differences between the mean scores at each visit across the 4 time intervals of the day, two-way ANOVA statistical analysis was used.

\section{Results}

In our study, ischemic stroke onset had the highest frequency in the 06.01-12.00 interval, followed by the 12.01-18.00 interval (Figs. 1 and 2), and the lowest frequency in the 00.01-06.00 interval. Patients were divided into two age groups ( $<65$ years, $\geq 65$ years $)-$ Fig. 3, with the predominance of patients aged less than 65 years in the morning interval. Figures 4 and 5 show the descriptive evolution of the arithmetic mean of NIHSS and mRS scores across the 4 time intervals of the day during the 2 years ( 5 evaluations).

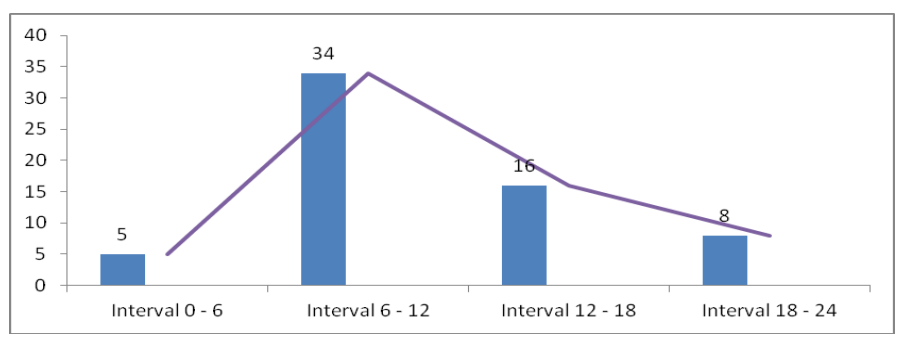

Fig. 1. Circadian variation of ischemic stroke onset in the studied group across the 4 time intervals of the day

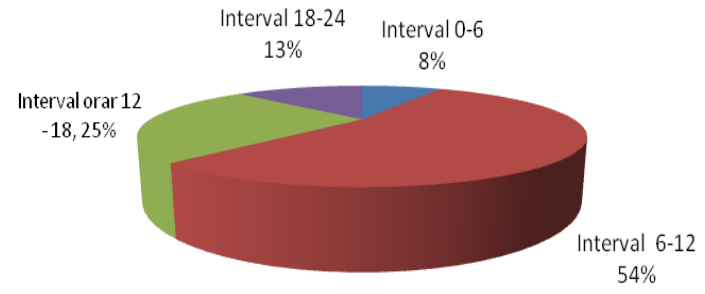

Fig. 2. Percentage representation of ischemic stroke onset in the studied group across the 4 time intervals of the day

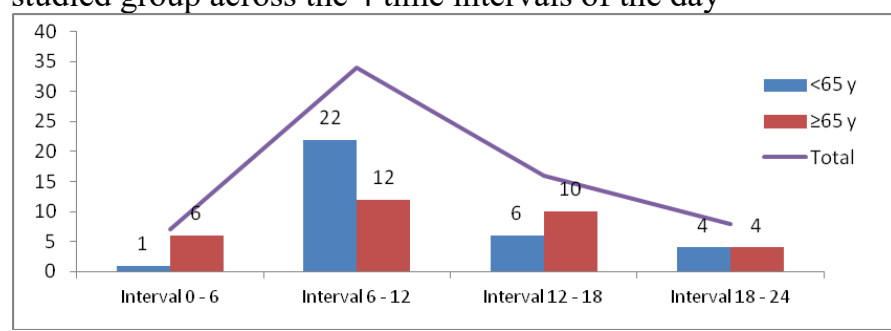

Fig. 3. Circadian variation of ischemic stroke onset in the studied group depending on age, across the 4 time intervals of the day

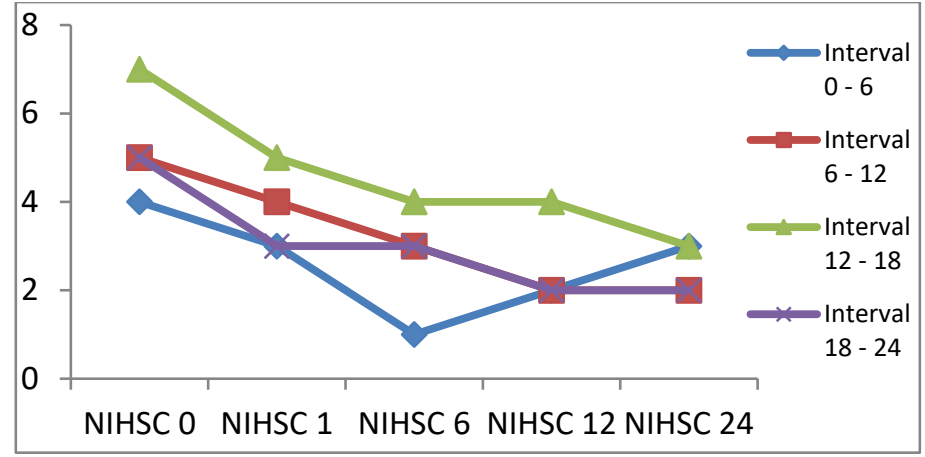

Fig. 4. Temporal evolution of the arithmetic mean of NIHSS scores over 2 years, across the 4 time intervals of the day ( 5 evaluations)

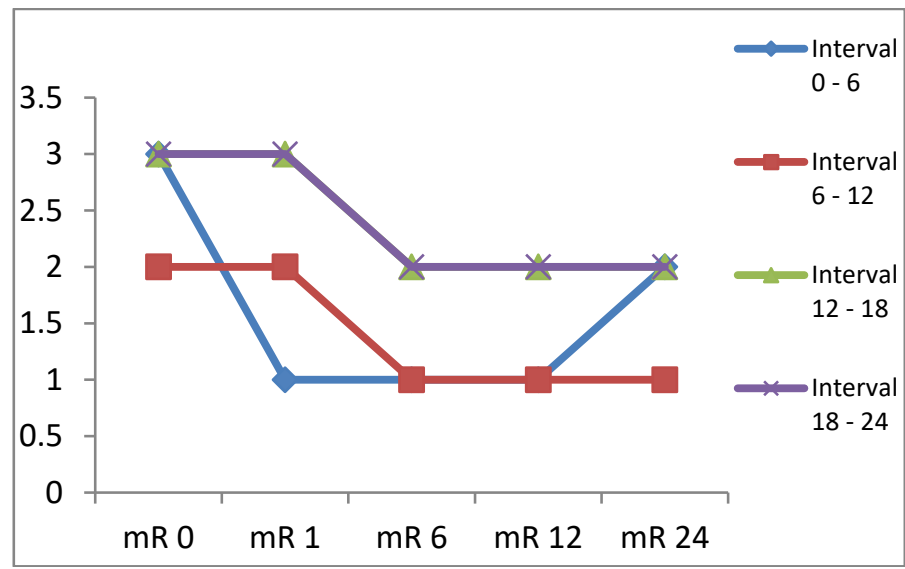

Fig. 5. Temporal evolution of the arithmetic mean of $\mathbf{m R S}$ scores over 2 years, across the 4 time intervals of the day (5 evaluations)

Following one-way ANOVA statistical analysis, statistically significant differences were found regarding patients' age (descriptively represented in Fig. 2) and the time interval of stroke onset (F $(3.59)=3.18$ (Welch), $p=0.011$ ), but the relationship between age and the stroke occurrence interval was of low intensity $\left(\omega^{2}=0.12\right)$. Post-hoc data analysis evidenced a significant difference between the 06.0112.00 and 00.01-06.00 intervals.

Two-way ANOVA statistical processing of NIHSS and $\mathrm{mRS}$ scores across the 4 time intervals of the day for the 5 evaluations $(0,1,6,12,24$ months) showed the following: no statistically significant differences were observed for NIHSS (but for NIHSS 6, $\mathrm{p}=0.073$ ), while for $\mathrm{mRS}$ score, statistically significant differences were found for mRS1 (F $(3.55)=5.6 \quad$ (Welch), $p=0.028)$. Post-hoc analysis indicated statistically significant differences between the 00.01-06.00 and 12.01-18.00 intervals, as well as between the 00.01-06.00 and 18.01-24.00 intervals. 


\section{Discussions}

In the case of the studied group, the circadian variation pattern of ischemic stroke is confirmed, having the highest incidence in the 06.01-12.00 interval and the lowest incidence during the night (00.01-06.00) (Fig. 1), according to literature data derived from both retrospective $(5-8,14)$ and prospective studies (15-17). This pattern is more obvious for patients aged less than 65 years.

By analyzing the diagrams for the evolution of NIHSS and mRS scores across the 5 evaluations during the 2 years of follow-up, it can be seen that NIHSS (reflecting the severity of the neurological picture) had a favorable evolution for all time intervals, excepting the nocturnal one (00.01-06.00), which was associated with its worsening after 6 months of resolution. This aspect was correlated with the evolution of the mean $\mathrm{mRS}$ score (reflecting the degree of disability or functional status), which also only for the nocturnal interval, after a rapid improvement by 2 points during the first month after stroke, remained unchanged for the rest of the first year, then started to worsen slowly by 1 point during the following 12 months. The highest mean NIHSS score at onset (NIHSS 0) was found for the 12.0118.00 interval (7), but its evolution was favorable (decreasing by 3 points during the first year), similarly to that of the 06.01-12.00 and 18.01-24.00 intervals $(5 \rightarrow 2)$. The greatest disability $(\mathrm{mRS})$ at onset was found for the 18.01-24.00 interval, along with the 00.01-06.00 interval mentioned before, but its evolution was favorable and similar to that of ischemic strokes with onset in the 06.01-12.00 and 12.01-18.00 intervals.

It can be concluded that these scores, reflecting clinical severity on the one hand and the degree of disability on the other hand, had an almost parallel evolution (Figs. 3 and 4).

Statistically significant differences between the 4 time intervals of stroke onset were found by multivariate ANOVA analysis only for mRS values at 1 month (mRS1), while for NIHSS the differences in values at 6 months (NIHSS 6) between the 4 time intervals of the day came close to statistical significance, most probably due to the small number of patients in the study group.

There are very few literature data on the evolution of NIHSS and mRS scores depending on the circadian interval in which stroke has occurred, the available data being derived only from the initial admission of patients with ischemic stroke (values at admission versus discharge), not from evolution during a longer follow-up period. However, the results are concordant, describing the best evolution of the functional score (mRS) from admission to discharge for patients with ischemic stroke onset in the 04.0108.00 interval and the lowest score in the 20.01-24.00 interval, without statistically significant differences for NIHSS $(8,11)$. The evolution of the mRS score is also consistent with the results of another study performed by us, in which the degree of disability was assessed based on ADL (activities of daily living) and IADL (instrumental activities of daily living) scores, with their least favorable evolution for ischemic stroke occurring in the nocturnal interval 00.01-06.00 and the greatest improvement for all time intervals during the first year after stroke (18).

\section{Conclusions}

This study confirms the circadian variation pattern for stroke of ischemic etiology, with the highest incidence in the morning and the lowest incidence during the night, our results being statistically significant for patients aged less than 65 years.

The evolution of NIHSS (reflecting the severity of the neurological picture in stroke) and mRS (reflecting the degree of disability) scores during the 2 years of follow-up, depending on the circadian interval of stroke onset, is similar for our study group, with the greatest improvement during the first year after stroke and relatively no changes during the second year, except for onset in the nocturnal interval (00.0106.00), associated with a slight worsening after 12 months. The difference in the evolution of the functional status of patients with nocturnal stroke onset compared to patients with onset in the other time intervals of the day is statistically significant for the evaluation visit at 1 month.

Knowing the influence of the circadian variation in ischemic stroke onset on the evolution of the neurological and functional picture can be important in assessing long-term prognosis, which involves different degrees of use of medical and social resources.

\section{Informed consent}

An informed consent was obtained from the patients included in this study.

\section{Declaration of conflict of interests}

The authors declare that there is no conflict of interest regarding the publication of this paper. 


\section{References}

1. Kelly-Hayes M, Wolf PA, Kase CS, Brand FN, McGuirk JM, D'Agostino RB. Temporal Patterns of Stroke Onset. Stroke. 1995;26(8):1343-7.

2. Wang H, Sekine M, Chen X, Kagamimori S. A study of weekly and seasonal variation of stroke onset. International journal of biometeorology. 2002;47(1):13-20.

3. Fodor DM, Perju-Dumbravã L. The circaseptan variation of stroke onset - a hospital-based study. Balneo Research Journal. 2018;9(4):414-7.

4. Fodor DM, Fodor M, Perju-Dumbravă L. Seasonal variation of stroke occurrence: a hospital based-study. Balneo Research Journal. 2018;9(2):82-7.

5. Manfredini R, Boari B, Smolensky MH, Salmi R, la Cecilia O, Maria Malagoni A, et al. Circadian Variation in Stroke Onset: Identical Temporal Pattern in Ischemic and Hemorrhagic Events. Chronobiology International. 2009;22(3):41753.

6. Turin TC, Kita Y, Rumana N, Nakamura Y, Takashima N, Ichikawa $\mathrm{M}$, et al. Is there any circadian variation consequence on acute case fatality of stroke? Takashima Stroke Registry, Japan (1990-2003). Acta Neurol Scand. 2012;125(3):206-12.

7. Fodor DM G-ND, Perju-Dumbrava L. Circadian patterns of ischemic stroke onset. HVM Bioflux. 2014;6(3):132-9.

8. Ripamonti L, Riva R, Maioli F, Zenesini C, Procaccianti G. Daily Variation in the Occurrence of Different Subtypes of Stroke. Stroke research and treatment. 2017;2017:9091250.

9. Beker MC, Caglayan B, Yalcin E, Caglayan AB, Turkseven S, Gurel B, et al. Time-of-Day Dependent Neuronal Injury After Ischemic Stroke: Implication of Circadian Clock Transcriptional Factor Bmall and Survival Kinase AKT. Molecular neurobiology. 2018;55(3):2565-76.

10. Ghandehari K. Challenging comparison of stroke scales. Journal of research in medical sciences : the official journal of Isfahan University of Medical Sciences. 2013;18(10):906-10.

11. Liou LM, Lin HF, Tsai CL, Lin RT, Lai CL. Timing of stroke onset determines discharge- functional status but not stroke severity: a hospital-based study. The Kaohsiung journal of medical sciences. 2013;29(1):32-6.

12. Jimenez-Conde J, Ois A, Rodriguez-Campello A, Gomis M, Roquer J. Does sleep protect against ischemic stroke? Less frequent ischemic strokes but more severe ones. Journal of neurology. 2007;254(6):782-8.

13. Hepburn M, Bollu PC, French B, Sahota P. Sleep Medicine: Stroke and Sleep. Missouri medicine. 2018;115(6):527-32.

14. Fodor DM, Babiciu I, Perju-Dumbrava L. Circadian Variation of Stroke Onset: A HospitalBased Study. Clujul Med. 2014;87(4):242-9.

15. Gupta A, Shetty H. Circadian variation in stroke - a prospective hospital-based study. Int J Clin Pract. 2005;59(11):1272-5.

16. Uddin MS, Hoque MI, Uddin MK, Kamol SA, Chowdhury RH. Circadian rhythm of onset of stroke - in 50 cases of ischemic stroke. Mymensingh medical journal : MMJ. 2015;24(1):121-6.

17. Shubham G. A study of clinical profile of stroke in a tertiary care hospital in Rajasthan. PARIPEX - Indian Journal of Research. 2017;6(6):59-61.

18. Fodor DM, Stănescu IC, Perju-Dumbravă L. The evolution of disability after ischemic stroke depending on the circadian variation of stroke onset. Balneo Research Journal. 2018;9(4):4113. 